\title{
Gestión estratégica de los recursos humanos y su relación con el desarrollo organizacional en la estación de servicios Unión en Trujillo, Perú
}

\section{Strategic management of human resources and its relationship with organizational development at the Unión Service Station in Trujillo, Peru}

José Germán Salinas-Gamboa; Ricardo Paz-Muñoz;

María Teresa Macedo-Mendoza³; María Chávez-Barbarán

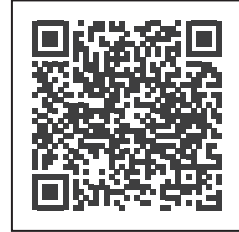

\section{Palabras clave:}

desarrollo de habilidades; estrategia de la investigación; gestión; Perú; recursos humanos; servicios.

Artículo de investigación

Fecha de recepción:

09 de diciembre de 2020

Fecha de aprobación:

06 de octubre de 2021

Fecha de publicación:

22 de noviembre de 2021

Esta publicación se encuentra bajo licencia:

Creative Commons

Reconocimiento-

NoComercial-SinObraDerivada 4.0 Internacional

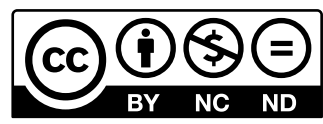

\section{Resumen}

Problemática: en la actualidad, está comprobado que el elemento humano es lo más valioso en las organizaciones debido a los constantes cambios y a la evolución en los negocios, de tal manera que valorar y hacer crecer a los trabajadores es primordial para que los negocios prevalezcan y sea un "ganar ganar". Objetivos: la presente investigación tiene como objetivo determinar la relación que existe entre la gestión estratégica de los recursos humanos y el desarrollo organizacional en la estación de servicios Unión de la ciudad de Trujillo, Perú, en 2020. Materiales y métodos: el tipo de estudio es relacional, con una muestra de 16 encuestados. Se aplicaron dos cuestionarios validados por el juicio

1 Licenciado, magíster y doctor en Administración. Licenciado y magíster en Educación. Responsable de la red de investigadores en Administración de América Latina y el Caribe. Perú. germansalinas@yahoo.es, ORCID: https:// orcid.org/0000-0002-8491-0751

2 Contador público y auditor. Maestro en Contribuciones. Doctorante en Finanzas, Facultad de Contaduría Pública de la Benemérita Universidad Autónoma de Puebla, México.ricardo.paz@correo.buap.mx; ricardo.paz@viep. com.mx. ORCID: https://orcid.org/0000-0002-1713-692X

3 Doctorante en Epistemología de la Investigación. Maestra en Administración de Pequeñas y Medianas Empresas (PYMES). Profesora investigadora de la Facultad de Contaduría Pública de la BUAP. México. teresa.macedo@correo. buap.mx. ORCID: https://orcid.org/0000-0001-9503-7065

4 Magíster en Talento Humano, Universidad Privada Antenor Orrego, Perú. mariachavez8@hotmail.com ORCID: https://orcid.org/0000-0001-6601-3582 


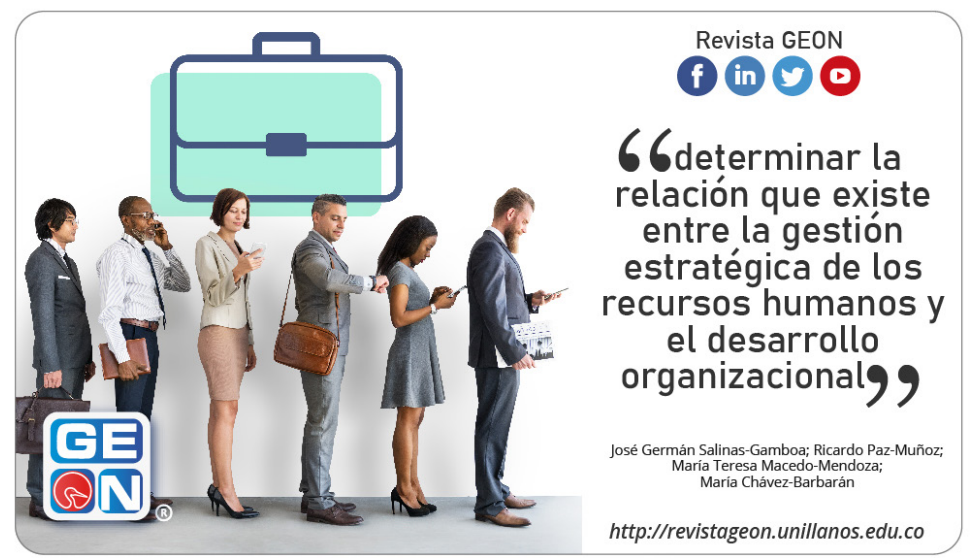

de expertos. Para contrastar las hipótesis, se utilizó el diseño preexperimental porque se trabajó con dos variables: gestión estratégica de los recursos humanos y desarrollo organizacional, aplicándose la prueba estadística de chi cuadrado para determinar la influencia entre las variables, cuyo valor de $p$ es $<0,05$. Resultados: la relación entre ambas variables es significativa, lo que constata la hipótesis formulada. Conclusiones: se concluye que la gestión estratégica de los recursos humanos se relaciona con el desarrollo organizacional en la estación de servicios Unión de la ciudad de Trujillo, Perú, en 2020. Contribución: el presente trabajo busca que la utilización de la gestión estratégica muestre resultados de aplicación en cualquier ente, ya sea público o privado, y ayude al crecimiento tanto de los trabajadores como de las entidades.

Palabras clave: desarrollo de habilidades; estrategia de la investigación; gestión; Perú; recursos humanos; servicios.

Códigos JEL: 124 Capital humano, productividad en

Cómo citar este artículo / Toreference this article:

Salinas-Gamboa, J. G., PazMuñoz, R., Macedo-Mendoza, M. T. , \& Chávez-Barbaran, M. (2021). Gestión estratégica de los recursos humanos y su relación con el desarrollo organizacional en la estación de servicios Unión en Trujillo, Perú. Revista GEON (Gestión, Organizaciones Y Negocios), 8(2), e-296. https://doi. org/10.22579/23463910.296 el trabajo.

\section{Abstract}

Problematic: At present it is proven that the human element is the most valuable in organizations due to the constant changes and evolution in business in such a way that valuing and making workers grow is essential for business to prevail and be a winwin. Objectives: The objective of this research is to 
Cómo citar este artículo /

Toreference this article:

Salinas-Gamboa, J. G., PazMuñoz, R., Macedo-Mendoza, M. T. ., \& Chávez-Barbaran, M (2021). Gestión estratégica de los recursos humanos y su relación con el desarrollo organizacional en la estación de servicios Unión en Trujillo, Perú. Revista GEON (Gestión, Organizaciones Y Negocios), 8(2), e-296. https://doi. org/10.22579/23463910.296 determine the relationship between the strategic management of human resources and its relationship with organizational development at the "Unión" service station in the city of Trujillo, Peru 2020. Materials and methods: The type of study is relational, being the sample of 16 respondents, applying two questionnaires validated by expert judgment and to contrast the hypothesis the pre-experimental design was used because it worked with two variables strategic management of human resources and organizational development, applying the statistical test of Chi square to determine the influence between the variables being its value $p<0.05$. Results: The relationship between both variables is significant, confirming the hypothesis formulated. Conclusions: Concluding that the strategic management of human resources is related to the organizational development in the Union Service Station of the city of Trujillo, Peru 2020. Contribution: this work seeks that the use of strategic management shows results of application in any entity, whether public or private, and helps the growth of both workers and entities.

Keywords: human resources; management; Peru; research strategy; services; skills development.

JEL Codes: J24 Human capital, productivity at work.

\section{Introducción}

En la actualidad, vivimos en un mundo de constantes cambios y solo aquellas organizaciones en las que el talento humano es considerado el recurso más valioso pueden alcanzar la competitividad en el mercado, ya que conocen que el capital humano ha evolucionado a través del tiempo. En este sentido, es importante hacer notar que, en el último cuarto del siglo $X X$, el recurso humano ha desarrollado fuerzas productivas en las áreas de la tecnología y la comunicación, así como competencias laborales (Valenzuela Salazar et al, 2020). Por consiguiente, la forma organizacional que ejerce en las empresas y la capacidad de adaptarse a nuevos escenarios de trabajo donde desarrolla diversas competencias, como el uso de la tecnología o el trabajo grupal y asincrónico, lo llevan a ejercer cada día más un papel de gran responsabilidad y a influir en los resultados positivos para la organización. 
Cada vez es más difícil dirigir al personal de una empresa debido a que las personas tienen diversas actitudes, motivaciones, percepciones, estilos de vida, sentimientos, entre otros, por lo que a todos no se les debería tratar por igual. Además, se debe mencionar que muchos gerentes toman decisiones concernientes a los recursos humanos con el fin de tomar acciones para llevar a bien los procesos de la empresa que, justamente, son gestionados por los colaboradores.

Por otro lado, el tema del desarrollo organizacional se ha vuelto importante hoy en día porque las empresas cada vez desean mejorar más. Esto implica la mejora en su personal y en la infraestructura, que debe ser moderna y estar diseñada de acuerdo con las necesidades de los clientes internos y externos. Por último, el desarrollo organizacional es una ventaja competitiva para muchas empresas. Además, la tecnología es aplicada por las empresas grandes y poderosas económicamente.

Para algunos autores, la administración de los recursos humanos se considera un tema de gran importancia porque las personas, llamadas también activos intelectuales, activos fijos, capital intelectual, entre otros, constituyen aquello que hace la diferencia entre las empresas competitivas y las que no lo son.

El desarrollo organizacional apareció en los años 60 y su principal impulsor fue Richard Beckhard. Se creó para que las organizaciones mejoren en todo aspecto, desde la parte de tecnología e infraestructura hasta las personas (Grieves, 2003, citado en Guízar-Montúfar, 2013).

Warren (1966, citado en Guízar-Montúfar, 2013) señala la importancia del desarrollo organizacional en todos los aspectos de la organización. Hospinal (2020) indica que las empresas que están apostando al cambio de forma constante, como parte de su estrategia, están logrando buenos resultados, por ende, algunas de ellas han aumentado su productividad en el 43 $\%$ y su creatividad en el $86 \%$; sin embargo, los buenos resultados se pueden ver reflejados en el $100 \%$ de los colaboradores comprometidos. Así mismo, Arney (2017) señala que hoy en día es importante la tecnología en las empresas, puesto que fomenta la calidad de los procesos.

Si analizamos la relación entre las dos variables (gestión estratégica de los recursos humanos y desarrollo organizacional), podemos decir que ambas están muy relacionadas porque la gestión estratégica contribuye a que las empresas mejoren sus procesos relacionados con la tecnología, la estructura y las personas. Este último aspecto se logra a través de una adecuada gestión que motive, incentive y ayude a que los colaboradores realicen mejor su trabajo.

En la empresa donde se realizó la investigación, la gran mayoría de las personas que trabajan allí se conoce de hace mucho tiempo. Por diversas razones que afectan los tiempos de entrega del servicio, muchos colaboradores se ausentan, piden cons- 
tantemente permiso o se enferman y piden licencia médica. Se presume que estas situaciones se propician para evitar ir al trabajo. Así mismo, existen diversas razones que impiden que se realice una correcta gestión del personal si no se emplean estrategias que conlleven a mejorar las diversas actividades o acciones en un futuro.

El propósito es estudiar cómo la gestión estratégica del recurso humano influye en el desarrollo organizacional de la estación de servicios Unión, en la ciudad de Trujillo, Perú, en 2020. Esta es considerada una empresa unipersonal, es decir, que tiene un solo dueño, es pequeña, se encuentra en el rubro de hidrocarburos y su capital es reducido. El estudio de estas variables es importante porque la gestión del personal, de los colaboradores o estratégica del recurso humano, como la llamaremos en esta ocasión, influye en la variable del desarrollo organizacional. A pesar de que este es utilizado correctamente por los gerentes, queremos estudiar si influye o no en este tipo de empresas.

Como antecedentes tenemos a Flores-Zambrano (2015), quien señala que es importante que los trabajadores de la organización sean personas que quieran desarrollarse permanentemente; por ende, cada vez se hace más importante la gestión del talento humano en las organizaciones lucrativas o no lucrativas.

Hernández-Barraza (2017) llegó a la conclusión de que siempre es bueno e importante diseñar estrategias para afrontar los cambios mundiales en las empresas. Vega-Franco y Álvarez-Escalante (2015) concluyeron que siempre es importante el bienestar de los colaboradores para que ellos se sientan contentos y con ganas de trabajar en la empresa. Así mismo, Pajuelo-Iglesias (2018) concluyó que cuanto mejor sean las habilidades gerenciales, mejor será el desarrollo organizacional.

Oblitas-Núñez (2018) concluyó que el desarrollo del plan estratégico institucional se basa especialmente en la parte de los recursos humanos, que son lo más importante en las instituciones públicas. Sánchez-Medina (2017) concluyó que sí se puede hacer desarrollo organizacional en todo tipo de empresas, sean estas con fines o sin fines de lucro.

Ante todo ello, planteamos el siguiente problema: ¿cómo la gestión estratégica de los recursos humanos se relaciona con el desarrollo organizacional de la estación de servicios Unión de la ciudad de Trujillo en 2020?, para lo cual tenemos las hipótesis que se presentan a continuación:

- Hi: existe una relación positiva y significativa entre la gestión estratégica de los recursos humanos y el desarrollo organizacional de la estación de servicios Unión de la ciudad de Trujillo en 2020.

- Ho: no existe una relación positiva y significativa entre la gestión estratégica de los recursos humanos y el desarrollo organizacional de la estación de servicios Unión de la ciudad de Trujillo en 2020. 
La presente investigación se justifica y es importante porque contribuye a que las empresas, como la estación de servicio Unión, gestionen a sus colaboradores de forma eficaz y eficiente, al tiempo que promueven el desarrollo organizacional, es decir, el desarrollo de toda la organización. Y, finalmente, contribuye a que las empresas puedan aplicar estos temas o conceptos con el objetivo de mejorar su relación con los clientes internos y externos, por medio de una adecuada gestión de los recursos humanos.

\section{Marco teórico}

Snell y Bohlander (2013) señalan que la administración de recursos humanos (ARH) es la utilización de los recursos humanos para alcanzar objetivos organizacionales. Igualmente, opinan que las funciones de la ARH se desarrollan y se trabajan mediante un sistema de administración de recursos humanos. Para los autores existen seis áreas funcionales que están asociadas con la administración efectiva de los recursos humanos: planeación de los recursos humanos, reclutamiento y selección, desarrollo de los recursos humanos, compensaciones y prestaciones, seguridad e higiene, relaciones laborales con los empleados e investigación de recursos humanos.

\section{Actividades de administración de recursos humanos}

Con respecto a las actividades de recursos humanos, Chiavenato (2011) sostiene que son las acciones relacionadas con la gestión de las personas.
Administrador de recursos humanos: un individuo que normalmente se desempeña en funciones de asesoría o de apoyo, trabajando con otros gerentes para ayudarlos a manejar asuntos de recursos humanos (Snell \& Bohlander, 2013).

Gestión: es un proceso de planeamiento, organización, dirección y control de diversas actividades, con el fin de lograr un objetivo (Strauss, 1994).

Proceso: secuencia de etapas o procesos con un orden lógico (Aragón et al., 2003).

Recursos humanos: son las personas o colaboradores que trabajan en la organización (Delgado \& Ena, 2011).

Desarrollo organizacional: una serie de acciones que tienen pasos paulatinos y concretos, cuya meta es un mejoramiento cognitivo de la institución y la generación de una gran producción que se evidencia en el mercado (Chiavenato, 2011).

\section{Gestión estratégica de los recursos humanos}

Vera-Vílchez (2017) sustenta que una buena gestión de los recursos humanos es importante para todas las empresas porque las personas son lo más valioso dentro de ellas.

\section{Gestión de los recursos humanos}

Vieira-Vieira (2014) afirma que la gestión de los recursos humanos es un instrumento de gestión administrativa que incluye la admisión, selección, 
capacitación, reclutamiento, seguridad y salud ocupacional, auditoria de personal, entre otros.

\section{Desarrollo organizacional}

Guízar-Montúfar (2013) sostiene que el desarrollo organizacional se define como el conjunto de actividades que se planifica dentro de la institución, el cual es llevado a cabo en muchos casos por el gerente de la empresa.

\section{Dimensiones del desarrollo organizacional}

De acuerdo con la teoría de Guízar-Montúfar (2013), el desarrollo organizacional se refiere a un aglutinado número de actividades planificadas que se llevarán a cabo, bajo la responsabilidad de los directivos, cuyos objetivos son importantes para lograr la eficiencia, eficacia y efectividad.

\section{Valores y principios fundamentales del desarrollo organizacional}

Hernández-Palomino et al. (2011) señalan que el paradigma del desarrollo organizacional valora el crecimiento humano y organizacional, el proceso participativo y de colaboración y el espíritu de búsqueda, para lo cual se basa en ciertos valores. Conforme el tiempo pasa, y de acuerdo con los valores de cada uno, las actitudes cambian; los valores en que se basa el desarrollo organizacional son: respeto por la gente (Hodges, 2017), confianza y apoyo (Burke \& Noumair, 2016), confrontación y participación.

\section{Fases del programa de desarrollo organizacional}

Burke (1988, citado por Guízar-Montúfar, 2013) presenta las fases que deben seguirse en los programas de desarrollo organizacional: entrada, diagnóstico, retroalimentación, planificación del cambio, inversión y evaluación.

\section{Elementos de la cultura organizacional en el desarrollo organizacional}

Según Guízar-Montúfar (2013), existen elementos que toda empresa debe tener en cuenta para poder desarrollarse correctamente: misión, visión, objetivos, valores, normas, reglamentos, entre otros, los cuales son importantes para el normal funcionamiento de la empresa.

\section{Materiales y métodos}

El tipo de estudio es relacional porque se analiza la relación de ambas variables que, según la teoría estudiada, existe. Debido a que la población es pequeña, se tomarán a todos los colaboradores de la estación de servicios Unión, que en total son 16. Estos datos fueron proporcionados por la gerente de la empresa, actualizados al 31 de enero de 2020. La técnica comprende las formas y métodos que se utilizan con la finalidad de recolectar los datos y la información. Esta es indispensable en el proceso de la investigación científica, ya que integra la estructura que organiza la investigación. Con respecto a los instrumentos, utilizamos los siguientes: 
cuestionario para medir la gestión estratégica de los recursos humanos y cuestionario para medir el desarrollo organizacional.

$\begin{array}{ll}\text { Variable } & \text { Instrumento } \\ \text { GERH } & \begin{array}{l}\text { Cuestionario de gestión } \\ \text { estratégica de los recursos } \\ \text { humanos }\end{array} \\ \text { DO } & \begin{array}{l}\text { Cuestionario para medir el } \\ \text { desarrollo organizacional }\end{array}\end{array}$

Para contrastar la hipótesis se utilizó el diseño preexperimental debido a que se trabajó con dos variables: gestión estratégica de los recursos humanos y desarrollo organizacional. Se aplicó la prueba estadística de chi cuadrado para determinar la relación causal de una variable sobre la otra. Los datos se procesaron y se analizaron mediante el programa estadístico informático SPSS versión 22.0, para determinar la relación causal entre las variables.

A partir de las dos variables se obtuvieron los resultados. Tanto la gestión estratégica de los recursos humanos como el desarrollo organizacional constan de 10 reactivos y cinco escalas ordinales. Así mismo, cabe señalar que los dos instrumentos (GERH y DO) se aplicaron el día 12 de marzo de 2020, teniendo como tiempo 1,5 horas en total. Ambos cuestionarios fueron realizados en las instalaciones de la estación de servicios Unión, en la ciudad de Trujillo. El perfil de los participantes para llevar a cabo las pruebas incluyó a todos los colaboradores de la empresa donde se hizo la investigación.
Objetivo 1: establecer el nivel de gestión estratégica de recursos humanos en la estación se servicios Unión, en 2020.

La tabla 1 nos muestra que la variable gestión estratégica de los recursos humanos es alta, con un $94 \%$. El 6 \% restante es considerado medio.

Tabla 1. Resultados de la gestión estratégica de los recursos humanos

\begin{tabular}{l|c|c}
\multicolumn{1}{c|}{ Niveles } & N & \% \\
\hline Alto & 15 & 94 \\
\hline Medio & 1 & 6 \\
\hline Bajo & 0 & 0 \\
Total & 16 & 100 \\
\hline
\end{tabular}

Fuente: datos obtenidos.

Escala: establecida por el instrumento de medición.

Objetivo 2: establecer el nivel de desarrollo organizacional en la estación de servicios Unión, en 2020.

En la tabla 2 se aprecia que el $88 \%$ de los encuestados señala que se realiza un desarrollo organizacional alto en la estación de servicios Unión, en 2020. Solo un $12 \%$ opina que es medio.

Tabla 2. Resultados del desarrollo organizacional

\begin{tabular}{l|c|c}
\multicolumn{1}{c|}{ Niveles } & N & \% \\
\hline Alto & 14 & 88 \\
\hline Medio & 2 & 12 \\
\hline Baja & 0 & 0 \\
Total & 16 & 100 \\
\hline
\end{tabular}

Fuente: datos obtenidos.

Escala: establecida por el instrumento de medición.

Objetivo 3: establecer el nivel de relación de la gestión estratégica de re- 
cursos humanos con cada dimensión de desarrollo organizacional en la estación de servicios Unión, en 2020.

En la tabla 3 se aprecia que la relación que existe entre la gestión estratégica de recursos humanos y la dimensión de incentivos es positiva moderada $($ Rho $=0,437)$.

En la tabla 4 se aprecia que la relación que existe entre la gestión estratégica de recursos humanos y la dimensión orientación al cambio es positiva moderada $($ Rho = 0,439).
Objetivo 4: establecer el nivel de relación del desarrollo organizacional con cada dimensión de la gestión estratégica de recursos humanos en la estación de servicios Unión, en 2020.

En la tabla 5 se aprecia la relación entre la dimensión gestión estratégica de recursos humanos con la variable desarrollo organizacional, cuyos resultados son los siguientes: las dimensiones factores sociales y orientación a resultados $(\mathrm{Rho}=0,356)$ y factores económicos y orientación sistemática (Rho $=0,282)$, con una correlación positiva moderada.

Tabla 3. Relación de la gestión estratégica de recursos humanos con la dimensión sistema de incentivos

\begin{tabular}{|c|c|c|c|c|}
\hline & & & Gestión E. R.H & Incentivos \\
\hline \multirow{6}{*}{ Rho de Spearman } & Gestión ERH & Coeficiente de correlación & 1,000 & $0,437 * \star$ \\
\hline & \multicolumn{2}{|r|}{ Sig. (bilateral) } & \multicolumn{2}{|r|}{0,000} \\
\hline & \multirow[b]{2}{*}{ Logro } & $\mathrm{N}$ & 16 & 16 \\
\hline & & Coeficiente de correlación & $0,437 * *$ & 1,000 \\
\hline & & Sig. (bilateral) & 0,000 & \\
\hline & & N & 16 & 16 \\
\hline
\end{tabular}

Fuente: elaboración propia.

Tabla 4. Relación de la gestión estratégica de recursos humanos con la dimensión orientación al cambio

\begin{tabular}{|c|c|c|c|c|}
\hline & & & Gestión E. R.H & Cambio \\
\hline \multirow{6}{*}{ Rho de Spearman } & Gestión ERH & Coeficiente de correlación & 1,000 & $0,439 * *$ \\
\hline & \multicolumn{2}{|r|}{ Sig. (bilateral) } & & 0,000 \\
\hline & \multicolumn{2}{|r|}{$\mathrm{N}$} & 16 & 16 \\
\hline & \multirow[t]{3}{*}{ Logro } & Coeficiente de correlación & $0,439 * *$ & 1,000 \\
\hline & & Sig. (bilateral) & 0,000 & \\
\hline & & N & 16 & 16 \\
\hline
\end{tabular}

Fuente: elaboración propia. 
Tabla 5. Relación de las dimensiones de gestión estratégica de recursos humanos y las dimensiones de desarrollo organizacional
Dimensiones
Gestión ERH
Desarrollo organizacional
Rho Spearman
Sig. bilateral

\begin{tabular}{|c|c|c|c|}
\hline \multirow{5}{*}{ Factores sociales } & Sistema de incentivos & $0,347 * *$ & 0,087 \\
\hline & Orientación a resultados & $0,356 * *$ & 0,289 \\
\hline & Orientación sistemática & $0,319 * \star$ & 0,088 \\
\hline & Orientación al cambio & 0,256 & 0,156 \\
\hline & & $0,508^{* *}$ & 0,000 \\
\hline \multirow{5}{*}{ Factores económicos } & Sistema de incentivos & $0,272 * \star$ & 0,013 \\
\hline & Orientación a resultados & $0,269 * *$ & 0,012 \\
\hline & Orientación sistemática & $0,282 * *$ & 0,010 \\
\hline & Orientación al cambio & 0,27 & 0,316 \\
\hline & & $0,408 *$ & 0,019 \\
\hline \multicolumn{4}{|c|}{$\begin{array}{l}\text { * La correlación es significativa en el nivel 0,05 (bilateral). } \\
\text { ** La correlación es significativa en el nivel 0,01 (bilateral). }\end{array}$} \\
\hline
\end{tabular}

Fuente: elaboración propia.

Objetivo general: establecer el nivel de relación de la gestión estratégica de recursos humanos con el desarrollo organizacional en la estación de servicios Unión en la ciudad de TrujiIlo, en 2020.

Para contrastar nuestras hipótesis empleamos la prueba de chi cuadrado, en donde:

Nivel de significancia será de $a=0,05$

Región de aceptación y rechazo:
RA/HO: valor de $p<0,05$

RR/HO: valor de $p>0,05$ (tabla 6)

Por tanto:

Al ser el valor de $p$ 0,000<0,05 aceptamos nuestra hipótesis alterna.

Hi: existe una relación positiva y significativa entre la gestión estratégica del recurso humano y el desarrollo organizacional en la estación de servicios Unión, en 2020.

Tabla 6. Prueba de chi cuadrado para constatar la hipótesis

\begin{tabular}{lllll} 
& Valor & df & Significación asintótica (bilateral) \\
\hline Chi-cuadrado de Pearson & $19,327 a$ & 6 & 0,000 \\
\hline Razón de verosimilitud & 26,411 & 6 & 0,000 \\
\hline Asociación lineal por lineal & 18,564 & 1 & 0,000 \\
\hline N de casos válidos & 16 & &
\end{tabular}

a. 6 casillas (50,0 \%) han esperado un recuento menor que 5. El recuento mínimo esperado es 0,04. 


\section{Discusión}

Durante el desarrollo de la investigación se fueron haciendo las coordinaciones para poder aplicar el instrumento el día de la realización de la prueba, con total transparencia y objetividad. Así mismo, hubo cierto temor por parte de algunos colaboradores, quienes estuvieron un poco reacios a la aplicación de la prueba porque dijeron no estar acostumbrados a ello.

En la tabla 2 se observa una alta gestión estratégica de los recursos humanos en la estación de servicios Unión, lo cual se relaciona con la tabla 3, en donde el resultado del desarrollo organizacional también es alto. En este sentido, Vera-Vílchez (2017) señala que la gestión de los recursos humanos debe responder hoy en día a la percepción de los empleados, manteniendo el equilibrio entre lo social y económico. Es allí donde entra a tallar el desarrollo organizacional de las empresas (Vieira-Vieira, 2014).

Por otro lado, la tabla 4 nos muestra una relación positiva moderada con cada una de las dimensiones de la variable desarrollo organizacional, es decir, con sistema de incentivos, orientación a resultados, orientación sistemática y orientación al cambio. Por ello, Snell y Bohlander (2013) señalan que el desarrollo del recurso humano debe darse durante la carrera que tiene en la empresa. Además, mencionan que las compensaciones, la seguridad e higiene laboral y las relaciones con los empleados son importantes.
Con respecto a la tabla 5, la relación entre las dos dimensiones de la gestión estratégica de los recursos humanos y las cuatro dimensiones del desarrollo organizacional en la estación de servicios, siendo las dimensiones factores sociales y orientación a resultados ( $\mathrm{Rho}=0,356)$, confirman lo dicho por Guízar-Montúfar (2013), quien señala que las estimulaciones apoyan el cumplimento de los objetivos, lo que se puede constatar en las dimensiones factores económicos y orientación sistemática ( Rho = 0,282). Además, se reafirma lo que dice Davis (1995, citado por Guízar-Montúfar, 2013), quien opina que es importante la interrelación entre gerentes y trabajadores. Por tanto, se obtiene una correlación significativa bilateral para ambos resultados.

Con respecto al objetivo general, en la tabla 6 la prueba de chi cuadrado nos permite ver que con un nivel de significancia de 0,05 se obtiene un valor de 0,27, lo cual nos indica una correlación positiva y significativa entre ambas variables. En este sentido, Flores-Zambrano (2015) señala que a través de la participación y el involucramiento en la empresa se hace importante la gestión estratégica del recurso humano, independiente de si la empresa es lucrativa o no. De este modo, se confirma la hipótesis planteada.

En consecuencia, se recomienda seguir realizando este tipo de estudios con dos variables y en los temas relacionados porque, si bien los resultados son positivos antes de la pandemia, sería importante e intere- 
sante hacer el estudio durante la pandemia, en donde muchas empresas han cerrado sus negocios, no específicamente las estaciones de servicio, pero la demanda de combustible ha sido menor debido a que las personas ya no viajan a diversos lugares por placer o trabajo, siendo importante analizar si la gestión de recursos humanos es la misma y si se puede dar desarrollo organizacional en épocas de pandemia en las empresas peruanas o internacionales. Además, es relevante un posible estudio posterior a la pandemia.

\section{Conclusiones}

La gestión estratégica del recurso humano que presentan las personas en la estación de servicios Unión en la ciudad de Trujillo, Perú, en 2020, es de nivel alto con un $94 \%$, como se muestra en la tabla 2. El desarrollo organizacional que presentan las personas en la estación de servicios, en 2020, es de nivel alto, con un 88 $\%$. Las dimensiones que presentan mayor relación son factores sociales y orientación a resultados (Rho $=0,356)$ y factores económicos y orientación sistemática (Rho = 0,282), con una correlación positiva moderada.

La relación entre la gestión estratégica del recurso humano y el desarrollo organizacional de las personas en la estación de servicios Unión, en 2020, es positiva y significativa dado que la prueba de chi cuadrado obtenida arrojó un valor de correlación de 0,27 y un valor de significancia de 0,0000. Dados los resultados, se considera que el personal que labora en la esta- ción se encuentra en correspondencia con la gestión estratégica y que, de alguna manera, sirve la aplicación de la metodología para cualquier otro negocio, donde se establezcan mediciones y se determine la satisfacción o no del personal dentro de la organización, teniendo en cuenta que esta es una herramienta que se puede aplicar a cualquier otra organización y en cualquier otro lugar.

\section{Información complementaria}

Agradecimientos: agradecemos la oportunidad de servir a través de la investigación a la Red Gestio por contribuir a este tipo de eventos y a nuestros familiares y amigos.

Contribuciones de autoría: el doctor José Germán Salinas Gamboa junto con la maestra María Chávez Barbarán llevaron a cabo la recopilación de la información básica de la estación de servicios Unión en la ciudad de Trujillo, Perú, así como las entrevistas y el armado de la investigación. Los maestros Ricardo Paz Muñoz y María Teresa Macedo Mendoza realizaron las correcciones y detalles, conforme a las bases de participación en el congreso. El maestro Ricardo Paz Muñoz hizo los ajustes a este documento para su aceptación y publicación en la revista Geon, siempre apoyado por los demás integrantes.

Conflictos de interés: el doctor José Germán Salinas Gamboa manifiesta que no existe conflicto de interés o circunstancia alguna que afecte la objetividad en la elaboración y presentación de este trabajo de investi- 
gación. El maestro Ricardo Paz Muñoz manifiesta que no existe conflicto de interés o circunstancia alguna que afecte la objetividad en la elaboración y presentación de este trabajo de investigación. La maestra María Teresa Macedo Mendoza manifiesta que no existe conflicto de interés o circunstancia alguna que afecte la objetividad en la elaboración y presentación de este trabajo de investigación. La maestra María Chávez Barbarán manifiesta que no existe conflicto de interés o circunstancia alguna que afecte la objetividad en la elaboración y presentación de este trabajo de investigación.

Financiamiento: el presente trabajo de investigación no recibió financiamiento alguno, ya que estuvo directamente a cargo de los investigadores participantes.

\section{Referencias}

Aragón, A., Fernández, M. L., Martín, F., Romero, P., Sánchez, G. \& Sanz, R. (2003). La Gestión Estratégica de los Re-

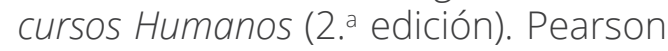
Educacoin.

Arney, E. (2017). Leraning for organizational Development. How to design, deliver and evaluate effective L\&D. Kogan Page. https://cutt.ly/RmFhwkg

Burke, W. W. \& Noumair, D. A. (2015). Organization Development. A Process of Learning and Chamging (third edition). Pearson Education. https://cutt. ly/3mFhioz

Chiavenato, I. (2011). Administración de recursos humanos. El capital humano de las organizaciones (9. edición). Mc Graw Hill.
Delgado, S. \& Ena, B. (2011). Recursos humanos. Ediciones Paraninfo Internacional.

Flores-Zambrano, S. G. (2015). Modelo estratégico de gestión de talento humano para la Superintendencia de Economía Popular y Solidaria [tesis de maestría, Pontificia Universidad Católica del Ecuador]. Repositorio Institucional PUCE. https://cutt.ly/8mFhsh4

Guízar-Montúfar, R. (2013). Desarrollo Organizacional. Principios y aplicaciones (4. ${ }^{a}$ edición). Mc Graw Hill Education.

Hernández-Barraza, A. (2017). Factores estratégicos que deben redefinir en su gestión los líderes de recursos humanos como socios del negocio para enfrentar los desafíos organizacionales que impone la economía digital [tesis de maestría, Universidad EAFIT]. Repositorio Institucional EAFIT. https://cutt.ly/XmFhgla

Hernández-Palomino, J. A., Gallarzo, M. \& Espinoza-Medina, J. J. (2011). Desarro/lo Organizacional (1. a edición). Pearson Educación.

Hodges, J. (2017). Consultancy, Organizational Development and Change. A practical guide to delivering value. Kogan Page. https://cutt.ly/VIWtpur

Hospinal, Jackeline (2020, enero 3). Employee experience: gestión clave para una empresa. Diario Gestión. Negocios. P. 6.

Oblitas-Núñez, B. (2018). Plan Estratégico Institucional y Gestión de Recursos Humanos de la Municipalidad de Lajas, Chota [tesis de maestría, Universidad César Vallejo]. Repositorio Institucional UCV. https://hdl.handle. net/20.500.12692/29052

Pajuelo-Iglesias, R. (2018). Habilidades Gerenciales y Desarrollo Organizacional 
desde la perspectiva de los trabajadores de la empresa Megamix Nijau S.A.C., San Juan de Lurigancho - 2018 [tesis de pregrado, Universidad César Vallejo]. Repositorio Institucional UCV. https:// hdl.handle.net/20.500.12692/33117

Sánchez-Medina, G. R. (2017). DesarroIlo organizacional y la asistencia a los recursos humanos en la Empresa Cruz del Sur - 2015 [tesis de maestría, Universidad César Vallejo]. Repositorio Institucional UCV. https://hdl.handle. net/20.500.12692/23764

Snell, S. \& Bohlader, G. (2013). Administración de Recursos Humanos (16. ${ }^{\text {a }}$ edición). Cengage Learning.

Strauss, G. (1994). Personal: Problemas Humanos en la Organización. Prentice Hall Iberia S.R.L.

Valenzuela Salazar, N. L., Martínez, C. P. B., Sánchez, V. L. V., \& Briones, C. T. R. (2020). Comunicación organizacional interna y su relación con la satisfacción de los empleados de una empresa concesionaria de automóviles de Piedras Negras Coahuila. Revista GEON (Gestión, Organizaciones Y Negocios), 7(1), 129-141. https://doi. org/10.22579/23463910.206
Vega-Franco, Á. M. \& Álvarez-Escalante, J. I. (2015). Diseño del plan estratégico de gestión humana con énfasis en bienestar social laboral para la Corporación Cultural y Deportiva del Comercio, Pereira 2015 [tesis de maestría, Universidad Tecnológica de Pereira]. Repositorio Institucional UTP. https://cutt.ly/PmFhkNm

Vera-Vílchez, M. E. (2017). Habilidades gerenciales y desarrollo organizacional del Departamento de Enfermería del Instituto Nacional de Salud del Niño, Lima 2016 [tesis de maestría, Universidad César Vallejo]. Repositorio Institucional UCV. https://hdl.handle. net/20.500.12692/9020

Vieira-Vieira, C. (2014). Gestión de recursos humanos: indicadores y herramientas. Observatorio Laboral Revista Venezolana. Universidad de Carabobo, 7(14), 23-33. http://servicio.bc.uc. edu.ve/faces/revista/lainet/lainetv7n14/art01.pdf 